\title{
Measurement of quality of life in pulmonary hypertension and its significance
}

\author{
E. Cenedese, R. Speich, L. Dorschner, S. Ulrich, M. Maggiorini, \\ R. Jenni and M. Fischler
}

ABSTRACT: Until recently, assessment of patients with pulmonary hypertension has mainly relied on functional and haemodynamic parameters. Health-related quality of life (HRQOL), however, has become increasingly important in defining overall health status.

The present study investigated the performance and clinical relevance of the Minnesota Living with Heart Failure (MLHF) questionnaire by prospectively studying 48 patients with either pulmonary arterial hypertension $(n=26)$ or chronic thromboembolic pulmonary hypertension $(n=22)$. The MLHF scores were correlated to various clinical and haemodynamic parameters. Prognostic outcome was evaluated by calculating the time taken to reach an adverse clinical event defined as death, lung transplantation or pulmonary endarterectomy.

The reliability of test-re-test and internal consistency of this HRQOL tool was high. The MLHF score and its physical subscore correlated moderately to well with functional and haemodynamic parameters, except in the case of pulmonary artery pressures. Both scores significantly improved during vasodilator therapy. This figure was surpassed only by the New York Heart Association/ World Health Organization functional class. A multivariate analysis of all variables revealed that the MLHF score was the sole factor predicting subsequent outcome.

The Minnesota Living with Heart Failure questionnaire is highly reproducible, consistent, and a moderately valid and responsive tool in assessing health-related quality of life in pulmonary hypertension. Moreover, it is a significant predictor of outcome in these patients.

KEYWORDS: Prognosis, pulmonary hypertension, quality of life, vasodilator therapy

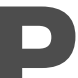

ulmonary hypertension (PH) comprises a group of uncommon disorders of the pulmonary circulation, characterised by a progressive increase in pulmonary arterial pressure (PAP), often leading to right heart failure and death [1]. After the exclusion of leftsided heart disease and chronic lung disorders, pulmonary arterial hypertension (PAH) and chronic thromboembolic pulmonary hypertension (CTEPH) represent the two main disease categories. Until recently, the prognosis of idiopathic $\mathrm{PAH}$ has been poor, with a median survival of $<3$ yrs [2]. However, since the widespread use of the various new treatment options, the long-term prognosis of PAH has significantly improved [3]. Although there are several reports on a potentially beneficial effect of vasodilator therapy in CTEPH, a recent randomised trial of inhaled iloprost found a significantly smaller response in these patients compared with those with PAH [4].

More than $20 \mathrm{yrs}$ ago, the first series of patients with PAH included primarily haemodynamic parameters as prognostic variables, such as mixed venous oxygen saturation $\left(\mathrm{S}_{\mathrm{v}}, \mathrm{O}_{2}\right)$ [5], right atrial and pulmonary artery pressures, and cardiac index [2]. Later, however, it became evident that baseline haemodynamic variables were poorly predictive, whereas functional aspects, such as New York Heart Association (NYHA) class and 6-min walking distance (6-MWD), had become the most relevant prognostic indicators [3].

In recent years, aspects of quality of life or, more specifically, health-related quality of life (HRQOL) have emerged as important clinical end-points, economic factors and, possibly, predictors of prognosis. However, only a few randomised trials in $\mathrm{PH}$ have included qualityof-life instruments [6]. Moreover, there are so far only sparse reports on the application, performance and potential prognostic value of HRQOL measurements in patients with $\mathrm{PH}$ [7-9]. Therefore, a prospective evaluation of the Minnesota Living with Heart Failure (MLHF) questionnaire was undertaken in a series of patients with $\mathrm{PH}$ with respect to its reproducibility, validity, responsiveness and prognostic significance.
AFFILIATIONS

Dept of Internal Medicine, University

Hospital, Zurich, Switzerland.

CORRESPONDENCE

R. Speich

Dept of Internal Medicine

University Hospital

Raemistrasse 100

CH-8091 Zurich

Switzerland

Fax: 4112554451

E-mail: klinspr@usz.unizh.ch

Received:

November 072005

Accepted after revision:

May 022006 


\section{METHODS}

\section{Patient selection}

From September 1999 to April 2003, patients with PH defined by a mean PAP $>25 \mathrm{mmHg}$ and a pulmonary artery occlusion pressure $\leqslant 15 \mathrm{mmHg}$ were consecutively assessed in accordance with internationally recommended guidelines [10]. Only patients with either PAH or CTEPH, as defined by the Revised Clinical Classification of Pulmonary Hypertension of Venice 2003 [1], were included in the study (table 1). Patients with left heart disease or significant obstructive or restrictive lung diseases were excluded $(n=7)$. In addition, four patients could not complete the questionnaire due to linguistic reasons. In total, 16 of the included patients had been participants of the Aerosolized Iloprost Randomized Study, a randomised, placebo-controlled trial of inhaled iloprost [4]. For the nine patients of the placebo group, baseline was defined as the endof-study date, when active treatment with inhalation of iloprost was started.

\section{Variables}

Quality of life was measured with a cross-cultural adaptation of the MLHF questionnaire [11] for German-speaking chronic heart failure patients [12]. The MLHF is a disease-specific, 21-item, self-administered tool measuring HRQOL in patients with heart failure. Patients rate their perceptions of how much their disease impacts the physical, socio-economic, and psychological aspects of daily life from 0 (not at all) to 5 (very much). For the present study, the term "heart failure" was replaced by "pulmonary hypertension". Scores on the total instrument range from 0 to 105, with higher scores reflecting a worse perceived quality of life. The first eight questions consider primarily physical functioning (physical subscore), whereas the last five pertain more to emotional aspects (emotional subscore).

The World Health Organization (WHO) classification of functional status of patients with $\mathrm{PH}$ [10], an adaptation of the NYHA functional classes, was applied using a standardised questionnaire. The 6-MWD was assessed according to the American Thoracic Society guidelines [13]. Immediately after exercise, the patients rated their baseline dyspnoea and overall fatigue using the Borg scale. Continuous-wave Doppler ultrasound was used to measure the velocity of the regurgitant jet of tricuspid regurgitation, and the peak pressure gradient between the right ventricle and right atrium was calculated by applying Bernoulli's equation $\left(4 v^{2}\right.$, where $v$ is the velocity of the regurgitant jet). This value is given as right ventricular systolic pressure without adding the estimated right atrial pressure. Invasive haemodynamic measurements were performed using standardised right heart catheterisation techniques [14]. Few patients were vasoreactive either by the old criteria (decrease in mean PAP of $>20 \% ; n=4$ ) or by the new criteria (decrease in mean PAP of $\geqslant 10 \mathrm{mmHg}$ to reach a value $\leqslant 40 \mathrm{mmHg} ; \mathrm{n}=4$ ). Three patients refused catheterisation, and in one case the procedure had to be interrupted early because of acute self-limited haemoptysis during wedging.

\section{Treatment and outcomes}

Patients were treated according to widely accepted evidencebased treatment algorithms [15]. All patients except one with a bleeding diathesis received oral anticoagulation (table 1).
Patients were treated predominantly with inhaled iloprost. As most of the patients were included into the study before the endothelin receptor antagonists became available, only six received bosentan. For continuous intravenous prostaglandin therapy $(n=6)$, iloprost was used instead of epoprostenol at the present authors' centre. Only two patients received monotherapy with calcium antagonists.

Patients were followed up at the present authors' $\mathrm{PH}$ outpatient clinic after 1, 3 and 6 months, and every 6 months thereafter. Beside clinical and laboratory evaluations, the MLHF score, NYHA/WHO functional class and 6-MWD were surveyed. Echocardiography was performed annually. Rightheart catheterisation was not routinely repeated. Prognosis was assessed by calculating the time taken to reach an adverse clinical event, which was defined as death, lung transplantation or pulmonary endarterectomy.

The latter operation was performed in three patients only, as, in Switzerland, the pulmonary endarterectomy programme is still in its infancy. Patients not reaching an adverse clinical event were censored at the time of the last follow-up. The mean follow-up time during the various vasodilator treatments was 12 months (95\% confidence interval (CI) 8-16).

\section{Statistical analysis}

Results are expressed as mean $\pm 95 \%$ CI. Cronbach's alpha coefficients were computed for the total MLHF score and its two subscores to evaluate internal consistency reliability [16]. The criterion of an alpha-value $>0.70$ was defined as evidence of satisfactory internal consistency reliability [17]. The reproducibility (test-re-test correlations), assessed by two postal surveys $\sim 1$ week apart on a separate group of 26 patients (16 PAH, $10 \mathrm{CTEPH}$ ), and the correlations between the various noninvasive and invasive parameters were determined using a bivariate analysis based on the Spearman's rank correlation. Time from inclusion in the study to an adverse clinical event was calculated using standard life-table analysis and KaplanMeier statistics. Potential predictors of outcome were assessed with the Cox univariate and multivariate proportional hazards analysis. Treatment effects were compared using the Wilcoxon's matched pairs test. The effect size was calculated according to COHEN [18] by dividing the difference between the mean values at baseline and at follow-up by the pooled SD of both values. Effect sizes are generally categorised as small (0.2), medium (0.5) and large (0.8). A p-value $\leqslant 0.05$ was considered to indicate statistical significance. With respect to multiple correlations (table 2), the strength of a Spearman's r-value of $0.01-0.20$ was arbitrarily considered to be low to absent, 0.20-0.39 weak, 0.40-0.59 moderate, 0.60-0.79 moderately strong, and $\geqslant 0.80$ very strong.

\section{RESULTS}

The baseline characteristics of the 48 study patients are shown in table 1. Two-thirds were female, and most were in NYHA/ WHO functional class III or IV (90\%). PAH was slightly more frequent than CTEPH. The mean 6-MWD was $362 \mathrm{~m} \mathrm{(95 \%} \mathrm{CI}$ 318-406), and the mean PAP was $51 \mathrm{mmHg}$ (95\% CI 46-56).

Ten patients died, four patients with $\mathrm{PAH}$ underwent lung transplantation and three with CTEPH underwent pulmonary endarterectomy. There was no significant difference in the time 


\begin{tabular}{|c|c|}
\hline \multicolumn{2}{|l|}{ Characteristic } \\
\hline Subjects $n$ & 48 \\
\hline Age yrs & $50(46-54)$ \\
\hline Sex (females) & $31(65)$ \\
\hline \multicolumn{2}{|l|}{ Diagnosis $n$} \\
\hline Pulmonary arterial hypertension & $26(54)$ \\
\hline Idiopathic & $18(38)$ \\
\hline Associated with collagen vascular disease & $3(6)$ \\
\hline Associated with Eisenmenger syndrome & $2(4)$ \\
\hline Associated with HIV infection & $3(6)$ \\
\hline Chronic thromboembolic pulmonary hypertension & $22(46)$ \\
\hline MLHF score at baseline & $49(43-55)$ \\
\hline Physical subscore & $24(21-27)$ \\
\hline Emotional subscore & $10(8-12)$ \\
\hline NYHA/WHO functional class & $3.3(3.1-3.5)$ \\
\hline Class II & $5(10)$ \\
\hline Class III & $22(46)$ \\
\hline Class IV & $21(44)$ \\
\hline 6-MWD m & $362(318-406)$ \\
\hline Borg scale & $4.2(3.5-4.9)$ \\
\hline Right ventricular systolic pressure gradient $\mathrm{mmHg}$ & $67(60-74)$ \\
\hline \multicolumn{2}{|l|}{ Invasive haemodynamics ${ }^{\#}$} \\
\hline Right atrial pressure $\mathrm{mmHg}$ & $9(7-11)$ \\
\hline Mean PAP mmHg & $51(46-56)$ \\
\hline Cardiac index $L \cdot \mathrm{m}^{-2}$ & $2.0(1.8-2.2)$ \\
\hline Mixed venous oxygen saturation \% & $58(55-61)$ \\
\hline \multicolumn{2}{|l|}{ Treatment } \\
\hline Oral anticoagulation & $47(98)$ \\
\hline Calcium antagonists (sole/combined therapy) & $2(4) / 14(29)$ \\
\hline Inhaled iloprost & $32(67)$ \\
\hline Bosentan & $6(13)$ \\
\hline Continuous intravenous iloprost & $6(13)$ \\
\hline Combination of inhaled iloprost and bosentan & $2(4)$ \\
\hline
\end{tabular}

Data are presented as mean (95\% confidence interval) or $\mathrm{n}(\%)$, unless otherwise stated. MLHF: Minnesota Living with Heart Failure; NYHA/WHO: New York Heart Association/World Health Organization; 6-MWD: 6-min walking distance; PAP: pulmonary arterial pressure. ${ }^{*}: \mathrm{n}=44.1 \mathrm{mmHg}=0.133 \mathrm{kPa}$.

taken to reach an adverse clinical event between patients with $\mathrm{PAH}$ and those with CTEPH (data not shown).

The test-re-test reliability of the MLHF score was $r=0.94$ for the total score, $r=0.93$ for the physical subscore, and $r=0.90$ for the emotional subscore (all $\mathrm{p}<0.001$ ).

The correlation matrix is shown in table 2. The correlations between the MLHF score and its subscore r-values were between 0.72 and 0.92 . Cronbach's alpha coefficient as a measure of internal consistency reliability was 0.92 for the total MLHF score and 0.88 for each of the two MLHF subscores.

As shown in tables 2 and 3, the correlations of the MLHF scores and the NYHA/WHO functional class, the Borg scale and the $\mathrm{Sv}_{\mathrm{v}} \mathrm{O}_{2}$ were moderate to moderately strong. Whereas the MLHF physical subscore correlated moderately with the NYHA/WHO functional class, the 6-MWD, the Borg scale, the

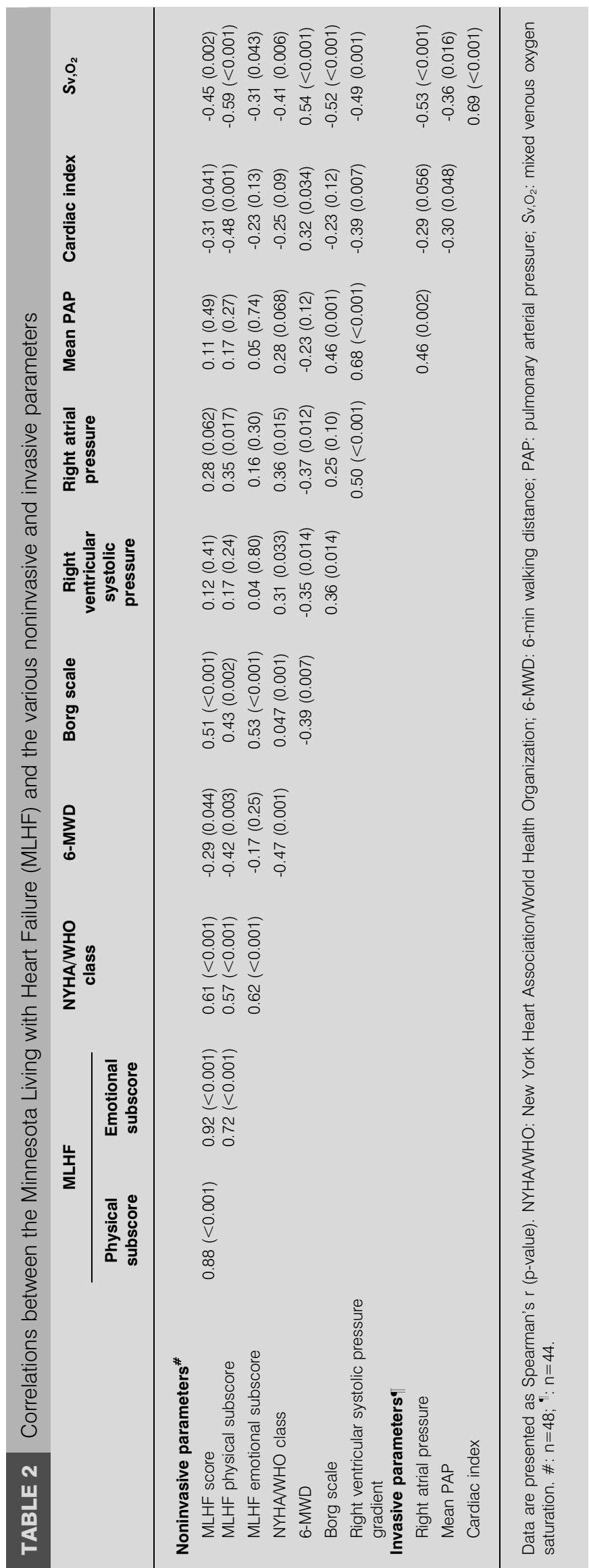


TABLE 3 Correlations between the Minnesota Living with Heart Failure (MLHF) and the various noninvasive and invasive parameters

\begin{tabular}{|c|c|c|c|}
\hline \multirow[t]{2}{*}{ Strength of correlation (range of $r$ ) } & \multicolumn{3}{|c|}{ MLHF } \\
\hline & Score & Physical subscore & Emotional subscore \\
\hline \multicolumn{4}{|l|}{ All patients ${ }^{\#}$} \\
\hline Little or none $(<0.20)$ & RVSP, RAP, mPAP & RVSP, mPAP & 6-MWD, RVSP, mPAP \\
\hline Weak $(0.20-0.39)$ & 6-MWD, cardiac index & RAP & Cardiac index, $\mathrm{Sv}, \mathrm{O}_{2}$ \\
\hline Moderate $(0.40-0.59)$ & Borg, $\mathrm{Sv}, \mathrm{O}_{2}$ & $\begin{array}{c}\text { NYHA, 6-MWD, Borg, cardiac index, } \\
\qquad{\mathrm{Sv}, \mathrm{O}_{2}}^{2}\end{array}$ & Borg \\
\hline Moderately strong (0.60-0.79) & NYHA & & NYHA \\
\hline \multicolumn{4}{|l|}{ PAH patients } \\
\hline Little or none $(<0.20)$ & RVSP, mPAP & RVSP, mPAP & RVSP, mPAP \\
\hline Weak $(0.20-0.39)$ & 6-MWD, Borg, cardiac index, $\mathrm{Sv}, \mathrm{O}_{2}$ & Borg & $\begin{array}{c}\text { 6-MWD, Borg, RAP, cardiac index } \\
{\mathrm{Sv}, \mathrm{O}_{2}}\end{array}$ \\
\hline $\begin{array}{l}\text { Moderate }(0.40-0.59) \\
\text { Moderately strong }(0.60-0.79)\end{array}$ & NYHA, RAP & $\begin{array}{c}\text { 6-MWD, RAP, cardiac index, } \mathrm{Sv}_{1} \mathrm{O}_{2} \\
\text { NYHA }\end{array}$ & NYHA \\
\hline \multicolumn{4}{|l|}{ CTEPH patients $^{+}$} \\
\hline Little or none $(<0.20)$ & RAP & & 6-MWD, RVSP, RAP \\
\hline Weak $(0.20-0.39)$ & 6-MWD, RVSP, mPAP & RVSP, mPAP & mPAP, cardiac index, $\mathrm{Sv}, \mathrm{O}_{2}$ \\
\hline Moderate $(0.40-0.59)$ & $\mathrm{NYHA}$, cardiac index, $\mathrm{Sv}, \mathrm{O}_{2}$ & $\begin{array}{c}\text { NYHA, 6-MWD, Borg, cardiac index, } \\
\text { Sv, } \mathrm{O}_{2}\end{array}$ & Borg \\
\hline Moderately strong $(0.60-0.79)$ & Borg & & NYHA \\
\hline
\end{tabular}

Data are presented as strength of correlation according to Spearman's $r$ (see Methods section). PAH: pulmonary arterial hypertension; CTEPH: chronic thromboembolic pulmonary hypertension RVSP: right ventricular systolic pressure over right atrial pressure; RAP: right atrial pressure; mPAP: mean pulmonary arterial pressure; 6-MWD: 6min walking distance; $\mathrm{Sv}, \mathrm{O}_{2}$ : mixed venous oxygen saturation; NYHA: New York Heart Association. ${ }^{\#}: \mathrm{n}=48 ;{ }^{\top}: \mathrm{n}=26 ;{ }^{+}: \mathrm{n}=22$.

TABLE 4 Effect size of repeatedly assessed parameters during vasodilator treatment

\begin{tabular}{lccc} 
& Change during treatment & p-value & Effect size \\
\hline MLHF score & $-10(-5--15)$ & $<0.001$ \\
MLHF physical subscore & $-5(-3--7)$ & $<0.001$ & -0.48 \\
MLHF emotional subscore & $-2(-1--4)$ & 0.011 & -0.54 \\
NYHA/WHO functional class & $-0.4(-0.2--0.7)$ & -0.38 \\
6-MWD m & $50(16-83)$ & -0.57 \\
Borg scale & $-0.6(0.3--1.6)$ & 0.001 & 0.10 \\
Right ventricular systolic pressure $\mathbf{m m H g}$ & $-6(2--14)$ & 0.13 & 0.27 \\
\hline
\end{tabular}

Data are presented as mean (95\% confidence interval), unless otherwise stated. MLHF: Minnesota Living with Heart Failure; NYHA/WHO: New York Health Association/ World Health Organization; 6-MWD: 6-min walking distance. $1 \mathrm{mmHg}=0.133 \mathrm{kPa}$.

cardiac index and the $\mathrm{S}_{\mathrm{v}, \mathrm{O}_{2}}$. The MLHF emotional subscore correlated only moderately with the Borg scale and moderately strongly with the NYHA/WHO functional class, respectively. For the other parameters, including the PAPs, the correlations were weak to absent. The correlations between the MLHF scores and the various variables with respect to the PAH and CTEPH subgroup of patients are shown in table 3. Remarkably, in contrast to the population as a whole, the right atrial pressure correlated moderately with the MLHF overall and the physical subscore in PAH patients.

In patients with NYHA/WHO functional class II, III and IV, the total MLHF score was 27 (95\% CI 9-45), 41 (34-48) and 62 (55-69), respectively ( $\mathrm{p}<0.001$ for class III versus IV).
The response to vasodilator treatment could be evaluated in 38 patients after a mean follow-up time of $1 \mathrm{yr}$. As shown in table 4, treatment improved (i.e. decreased) the MLHF scores significantly. The improvements in the overall score and the physical subscore had moderate effects of -0.48 and -0.54 , respectively. These values were surpassed only by those of the NYHA/WHO functional class (-0.57). The 6-MWD improved by $50 \mathrm{~m}$ (95\% CI 16-83). The respective effect, however, was rather low (0.32). The Borg scale and the right ventricular systolic pressure did not improve significantly.

In the univariate model assessment of the prognostic role of the various parameters, the MLHF score and the Borg scale were the sole predictive factors of the noninvasive variables 
TABLE 5 Univariate and multivariate analysis of noninvasive and invasive prognostic parameters

\begin{tabular}{|c|c|c|c|c|}
\hline & \multicolumn{2}{|c|}{ Univariate analysis } & \multicolumn{2}{|c|}{ Multivariate analysis } \\
\hline & Hazard ratio & p-value & Hazard ratio & p-value \\
\hline \multicolumn{5}{|l|}{ Noninvasive parameters ${ }^{\#}$} \\
\hline MLHF score at baseline per 10 points & $1.55(1.18-2.03)$ & 0.002 & $1.73(1.11-2.69)$ & 0.015 \\
\hline NYHAWHO functional class per class & $1.93(0.83-4.51)$ & 0.13 & $0.40(0.09-1.74)$ & 0.22 \\
\hline Right ventricular systolic pressure $10 \mathrm{mmHg}^{-1}$ & $1.02(0.82-1.30)$ & 0.87 & $0.77(0.54-1.11)$ & 0.16 \\
\hline \multicolumn{5}{|l|}{ Invasive parameters } \\
\hline Right atrial pressure $\mathrm{mmHg}^{-1}$ & $1.10(1.01-1.20)$ & 0.022 & $1.03(0.88-1.20)$ & 0.73 \\
\hline mPAP $10 \mathrm{mmHg}^{-1}$ & $1.22(0.93-1.62)$ & 0.16 & $1.22(0.81-1.83)$ & 0.34 \\
\hline Cardiac index $\mathrm{L} \cdot \mathrm{m}^{-2}$ & $0.15(0.04-0.62)$ & 0.009 & $0.94(0.13-6.64)$ & 0.95 \\
\hline Mixed venous oxygen saturation per $10 \%$ & $0.39(0.24-0.64)$ & 0.001 & $0.45(0.12-1.68)$ & 0.24 \\
\hline
\end{tabular}

Data are presented as mean (95\% confidence interval), unless otherwise stated. MLHF: Minnesota Living with Heart Failure; NYHA/WHO: New York Heart Association/ World Health Organization; 6-MWD: 6-min walking distance; mPAP: mean pulmonary arterial pressure. ${ }^{\#}: \mathrm{n}=48 ;{ }^{\bullet}: \mathrm{n}=44.1 \mathrm{mmHg}=0.133 \mathrm{kPa}$

(table 5). As in the correlative analysis, also with respect to prognosis, neither the right ventricular systolic pressures measured by Doppler echocardiography nor the mean pressure determined by right heart catheterisation had any prognostic significance. The other haemodynamic variables, however, had a significant prognostic role in the univariate model; the mixed venous saturation was the best predictor of adverse outcome.

When all the noninvasive and invasive parameters were introduced into a multivariate model, only the MLHF score remained significant $(\mathrm{p}=0.015)$. Patients with a MLHF score of $\leqslant 40$ had a significantly better overall outcome than those with a score of $>40$ (fig. 1). The respective survival curves were

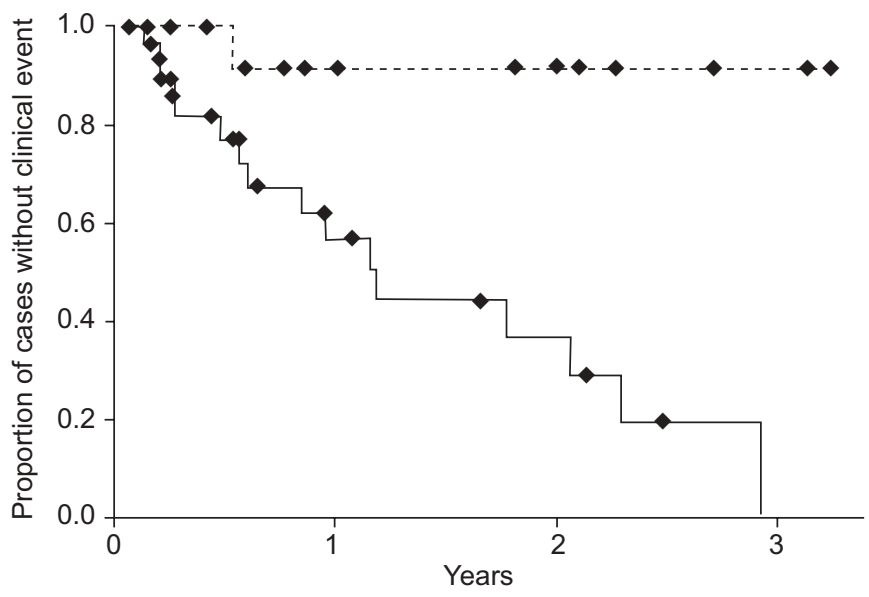

FIGURE 1. Kaplan-Meier estimates of likelihood of not reaching the endpoints of death, lung transplantation or pulmonary endarterectomy. Patients with a Minnesota Living with Heart Failure score of $\geqslant 40$ (- $\longrightarrow$ ) had a significantly worse prognosis than those with a score of $<40(------; p=0.0018$; multivariate analysis $p=0.015)$. comparable for PAH (the overall mortality was 14 versus $48 \%$ for a MLHF score of $<40$ versus $\geqslant 40$ ) and CTEPH patients (the overall mortality was 0 versus $57 \%$ for a MLHF score of $<40$ versus $\geqslant 40$ ). These differences, however, were not significant due to the low number of patients in each group.

\section{DISCUSSION}

The present study demonstrates a high test-re-test and internal consistency reliability, as well as a moderate validity and responsiveness of the MLHF questionnaire in the assessment of HRQOL in patients with $\mathrm{PH}$. Moreover, a multivariate analysis including various other noninvasive and invasive parameters of disease severity showed that the MLHF score remained the sole significant prognostic variable.

Currently, there are three papers specifically addressing quality of life in $\mathrm{PH}$ [7-9]. The Papworth group has recently designed a new $\mathrm{PH}$-specific tool with the acronym CAMPHOR (Cambridge Pulmonary Hypertension Outcome Review), which consists of the following three scales: 1) overall symptoms (made up of energy, breathlessness and mood subscales); 2) functioning; and 3) quality of life [7]. The CAMPHOR scales had good internal consistency (Cronbach's alpha $=0.90-0.92$ ) and reproducibility (test-re-test correlations $=0.86-0.92)$. SHAFAZAND et al. [8] used the general Nottingham Health Profile questionnaire and the diseasespecific Congestive Heart Failure Questionnaire in 53 patients with $\mathrm{PAH}$, and found moderate-to-severe impairments in all items compared with population normalities. Patients receiving epoprostenol reported more energy and had better emotional scores. TAICHMAN et al. [9] found a decrease in all HRQOL domains of the SF-36 ${ }^{\circledR}$ and of the respiratory-disease specific Saint George's Respiratory Questionnaire. The 6-MWD correlated better with HRQOL $(r=0.62)$, as did the NYHA/ WHO functional class. Haemodynamic measurements, however, showed no correlation with HRQOL.

In the present study, the reproducibility of the MLHF scores assessed by test-re-test correlations was $\geqslant 0.90$. These values 
were comparable with those of the CAMPHOR Study [7], as well as with those of the original study $(\geqslant 0.88)$ in patients with left heart failure, for whom the MLHF score was initially designed by RECTOR and COHN [19].

The reliability of internal consistency of the MLHF scores in the present authors' $\mathrm{PH}$ patients was good (Cronbach's alpha $\geqslant 0.88$ ), and thus comparable with the values found in the above-mentioned heart failure study [19], as well as in the CAMPHOR series [7]. However, it has to be mentioned that these high Cronbach's alpha values might also indicate some redundancy in the questions used in the MLHF questionnaire.

The validity of the MLHF questionnaire was assessed by correlating the MLHF scores with various noninvasive and invasive baseline clinical indicators of disease severity. There was a moderate-to-moderately strong correlation of all scores with the Borg scale and NYHA/WHO functional class, the latter being comparable with the respective values of the CAMPHOR score $(\mathrm{r}=0.57-0.62$ and $0.47-0.62$, respectively) [7]. The 6-MWD correlated only moderately with the MLHF physical subscore $(r=-0.42)$ This was comparable with the respective value of the quality-of-life subscale of the CAMPHOR score $(r=-0.41)$ [20], but lower than that of the overall symptom $(\mathrm{r}=-0.53)$ and functioning $(\mathrm{r}=-0.63)$ subscales of that score, as well as the SF-36 ( $\mathrm{r}=0.62)$ used by TAICHMAN et al. [9].

Most of the correlations in the present series were comparable with those of the MLHF scores with oxygen deficit, exercise duration and peak oxygen consumption, respectively, in left heart failure patients $(r=0.33-0.61)[11,19,21]$. In addition, the validity of the MLHF questionnaire in the present authors' $\mathrm{PH}$ patients is highlighted by its good discrimination between different stages of severity assessed by the NYHA/WHO functional class. The mean value of 41 (95\% CI 34-48) for NYHA/WHO class III was comparable with the values of patients with left heart failure in NYHA class II or III $(45 ; 95 \%$ CI 43-47) in the Studies of Left Ventricular Dysfunction (SOLVD) study [21].

Interestingly, apart from cardiac index $(\mathrm{r}=-0.48$ with physical subscore $)$ and the $S_{v}, \mathrm{O}_{2}(\mathrm{r}=-0.45$ and $\mathrm{r}=-0.59$ with overall score and physical subscore, respectively), in analogy to the findings of TAICHMAN et al. [9], the other haemodynamic parameters, especially PAPs, did not correlate with the MLHF scores. Historically, along with data from the National Institute of Health cohort [2], invasive haemodynamic measurements were used as a primary end-point in the first epoprostenol trial [15]. However, it soon became clear that resting haemodynamics were only loosely correlated with the clinical state, functional class, exercise capacity, response to therapy and prognosis. In this respect, CASTELAIN et al. [22] have shown that improvement in exercise tolerance during epoprostenol therapy is mainly ascribable to a decrease in the incremental pulmonary vascular resistance during exercise. Thus, determination of PAPs at rest may only poorly reflect maximum circulatory and functional capacity.

Responsiveness of the MLHF questionnaire was investigated by calculating the effect sizes of the changes in its scores during vasodilator therapy (table 4). The MLHF scores significantly improved (i.e. decreased) after a mean treatment period of $1 \mathrm{yr}$. Although the improvements in the overall score and physical subscore had only a moderate effect size, their responsiveness was surpassed only by the NYHA/WHO functional class. All other noninvasive measurements had a lower effect size. The Borg scale and right ventricular systolic pressure did not improve significantly during vasodilator therapy. The current authors think that the main reason for these only moderate effect size values is the relatively small number of patients in the present series, which results in rather high SDs and thus substantially influences the respective calculations [18]. A clue to this interpretation might be the improvement in the 6-MWD of $50 \mathrm{~m}(95 \%$ CI 16-83, $\mathrm{p}=0.001)$ in patients in the present study, which was comparable with figures in the large $\mathrm{PH}$ trials [15], but resulted in an effect size of only 0.32 . The fact that the changes in MLHF scores were comparable with those of the 6-MWD in larger treatment trials, but that the former resulted in a larger effect size, might be due to a higher precision of the MLHF score, as the effect size is calculated by dividing the difference between the mean values at baseline and at follow-up by the pooled SDs, i.e. the precision of both values [18].

The net improvements in HRQOL in the present study $(-10$; $95 \%$ CI -5- -15) were comparable with those in the two heart failure studies using the MLHF questionnaire, namely the enalapril (SOLVD) and the pimobendan trial, with improvements in overall score of -5 and -8 , respectively [21]. Notably, there was no improvement in HRQOL assessed by the MLHF questionnaire in six other left heart failure studies, as summarised by BERRY and MCMURRAY [21].

Examining the prognostic impact of the different variables, it was found that the MLHF score, the Borg scale and the haemodynamics, except the PAPs, were significant predictors of outcome in the univariate analysis. In the multivariate model, however, only the MLHF score remained as a significant prognostic factor. This finding is intriguing and difficult to explain. As reviewed by BENYAMINI and IDLER [23, $24]$, there are almost 50 studies that have examined selfperceived health and mortality in the general population, and most of them found that respondents with self-perceived poor health have a two to seven times higher mortality than subjects with self-perceived excellent health, even when all other medical data were taken into account. Several possible explanations for this phenomenon have been discussed, among them inadequate statistical controls for some variables, confounding factors, the influence of external resources, such as social contact and support, and internal resources, including general well-being, optimism, or religion $[25,26]$. In addition, IDLER and KASL [26] hypothesised that, first, the perception of one's own health status has some beneficial or detrimental effect on the individual's subsequent morbidity and mortality and, secondly, that there is a self-perceived area of variation in morbidity and mortality through the individual's inherent knowledge about "silent" biological risk factors, medical conditions or personal lifestyle.

With respect to HRQOL in specific medical conditions, there are several studies in patients with left heart diseases that are in line with the present series showing an independent significant correlation of HRQOL and subsequent prognosis, even in a setting of other covariates with high prognostic 
impact [21]. At least one of these series also included the MLHF questionnaire [27]. The investigators proposed that there may be a psychological state characterised by reduced vigour, lack of initiative and withdrawal from activities of daily living associated with an increased mortality, and that active engagement in daily life serves as a protective mechanism. In addition, it is conceivable that measurements of HRQOL reflect more globally various parameters of wellbeing or general health status than single variables, such as NYHA/WHO functional class, 6-MWD or haemodynamics. In addition, HRQOL may be affected by more than just the symptoms of a specific disease. For example, in the PRECISE trial there was no significant difference in the MLHF score between carvedilol and placebo, despite a significant increase in ejection fraction and a significant decrease in the combined risk of morbidity and mortality [21]. This was probably due to an increase in adverse reactions, including dizziness, fatigue, syncope and bradycardia, in the active treatment group. Thus, measurements of HRQOL assess a wide variety of physical, psychological and social parameters that are collated into an overall score reflecting general health probably more adequately than any single physiological variable. Moreover, a recent study found that in addition to cardiac index and acute vasoreactivity, low serum albumin and being of AfricanAmerican or Asian descent were independent predictors of outcome in PAH [28]. These findings add to the present authors' notion that so-far neglected or unknown biological or socio-economic factors may be crucial for disease-specific prognosis.

The MLHF questionnaire was included in the large treprostinil trial [29]. Although there are no precise data given, in contrast to the present series, only the physical subscore changed significantly in the 233 patients receiving active treatment $(\mathrm{p}=0.0064)$, possibly reflecting the overall poor average improvement in exercise capacity of only $16 \mathrm{~m}$ in this study. In the second, beraprost trial [30], in accordance with the absence of a clinical benefit, none of the MLHF scores was found to have improved. However, in patients with CTEPH, pulmonary endarterectomy resulted in an impressive improvement in HRQOL measured by the SF-36 [31]. Post-operatively, the patients scored only four out of eight composite health concepts slightly lower compared with the normal population.

The following limitations of the present study have to be mentioned. First, only a relatively modest number of patients could be included. However, the present authors think that in consideration of the fact that most of their present results are plausible, highly consistent and significant, a beta error is improbable. Secondly, the inclusion of patients with PAH and CTEPH might compromise the validity of the present study. However, both conditions share pathophysiological, clinical and possibly prognostic features. In addition, it has to be mentioned that in the CAMPHOR evaluation study, both groups of patients were included [7]. Thirdly, it has to be mentioned that the very low number of pulmonary endarterectomies in the present series, as well as the inclusion of this most often curative operation as an adverse clinical event, may be questionable, even though the operation carries a significant risk of mortality ranging from $5 \%$ in experienced centres up to $25 \%$ in some smaller series.
In conclusion, the present series demonstrate a high reproducibility and internal consistency reliability as well as a moderately good validity and responsiveness of the Minnesota Living with Heart Failure questionnaire in pulmonary hypertension, supporting its plausibility as a diseasetargeted assessment tool in these patients. Most interestingly, a multivariate analysis showed that the Minnesota Living with Heart Failure score remained as the sole prognostic parameter. Thus, health-related quality of life indices, such as the Minnesota Living with Heart Failure score, may provide additional clinical and prognostic information in patients with pulmonary hypertension.

\section{ACKNOWLEDGEMENTS}

The authors are indebted to J. Muntwyler for statistical advice, U. Treder for collecting the health-related quality of life data, and $\mathrm{H}$. Boeschenstein for proof reading the manuscript.

\section{REFERENCES}

1 Simonneau G, Galie N, Rubin LJ, et al. Clinical classification of pulmonary hypertension. J Am Coll Cardiol 2004; 43: Suppl. 12, 5S-12S.

2 D'Alonzo GE, Barst RJ, Ayres SM, et al. Survival in patients with primary pulmonary hypertension. Ann Intern Med 1991; 115: 343-355.

3 Sitbon O, Humbert $\mathrm{M}$, Nunes $\mathrm{H}$, et al. Long-term intravenous epoprostenol infusion in primary pulmonary hypertension: prognostic factors and survival. J Am Coll Cardiol 2002; 40: 780-788.

4 Olschewski H, Simonneau G, Galie N, et al. Inhaled iloprost for severe pulmonary hypertension. $N$ Engl J Med 2002; 347: 322-329.

5 Fuster V, Steele PM, Edwards WD, et al. Primary pulmonary hypertension: natural history and the importance of thrombosis. Circulation 1984; 70: 580-587.

6 Hoeper MM, Oudiz RJ, Peacock A, et al. End points and clinical trial designs in pulmonary arterial hypertension: clinical and regulatory perspectives. J Am Coll Cardiol 2004; 43: Suppl. 12, 48S-55S.

7 McKenna S, Doughty N, Meads D, et al. The Cambridge Pulmonary Hypertension Outcome Review (CAMPHOR): A measure of health-related quality of life and quality of life for patients with pulmonary hypertension. Qual Life Res 2006; 15: 103-115.

8 Shafazand S, Goldstein MK, Doyle RL, et al. Health-related quality of life in patients with pulmonary arterial hypertension. Chest 2004; 126: 1452-1459.

9 Taichman DB, Shin J, Hud L, et al. Health-related quality of life in patients with pulmonary arterial hypertension. Respir Res 2005; 6: 92.

10 Barst RJ, McGoon M, Torbicki A, et al. Diagnosis and differential assessment of pulmonary arterial hypertension. J Am Coll Cardiol 2004; 43: Suppl. 12, 40S-47S.

11 Rector TS, Kubo SH, Cohn JN. Patient self-assessment of their congestive heart failure part 2: content, reliability and validity of a new measure the Minnesota Living with Heart Failure Questionnaire. Heart Fail 1987; 3: 198-209.

12 Quittan M, Wiesinger GF, Crevenna R, et al. Cross-cultural adaptation of the Minnesota living with heart failure 
questionnaire for German-speaking patients. J Rehabil Med 2001; 33: 182-186.

13 American Thoracic Society Committee on Proficiency Standards for Clinical Pulmonary Function Laboratories, ATS statement: guidelines for the six-minute walk test. Am J Respir Crit Care Med 2002; 166: 111-117.

14 Chemla D, Castelain V, Herve P, et al. Haemodynamic evaluation of pulmonary hypertension. Eur Respir J 2002; 20: 1314-1331.

15 Galie N, Seeger W, Naeije R, et al. Comparative analysis of clinical trials and evidence-based treatment algorithm in pulmonary arterial hypertension. J Am Coll Cardiol 2004; 43: Suppl. 12, 81S-88S.

16 Cronbach LJ. Coefficient alpha and the internal structures of tests. Psychometrika 1951; 16: 297-334.

17 Bennet SJ, Oldridge NB, Eckert GJ, et al. Discriminant properties of commonly used quality of life measures in heart failure. Qual Life Res 2002; 11: 349-359.

18 Cohen J. Statistical power analysis for the behavioral sciences. 2nd Edn. Hillsdale, NJ, Lawrence Erlbaum, 1988.

19 Rector TS, Cohn JN. Assessment of patient outcome with the Minnesota Living with Heart Failure questionnaire: reliability and validity during a randomized, double-blind, placebo-controlled trial of pimobendan. Pimobendan Multicenter Research Group. Am Heart J 1992; 124: 1017-1025.

20 Doughty N, McKenna S, Meads D, et al. The CAMPHOR: correlations with objective measures of severity in pulmonary hypertension. Am J Respir Crit Care Med 2005; 171: A801.

21 Berry C, McMurray J. A review of quality-of-life evaluations in patients with congestive heart failure. Pharmacoeconomics 1999; 16: 247-271.

22 Castelain V, Chemla D, Humbert M, et al. Pulmonary artery pressure-flow relations after prostacyclin in primary pulmonary hypertension. Am J Respir Crit Care Med 2002; 165: 338-340.

23 Idler EL, Benyamini Y. Self-rated health and mortality: a review of twenty-seven community studies. J Health Soc Behav 1997; 38: 21-37.

24 Benyamini Y, Idler EL. Community studies reporting association between self-rated health and mortality; additional studies, 1995 to 1998. Res Aging 1999; 21: 392-401.

25 Contrada RJ, Goyal TM, Cather C, et al. Psychosocial factors in outcomes of heart surgery: the impact of religious involvement and depressive symptoms. Health Psychology 2004; 23: 227-238.

26 Idler EL, Kasl S. Health perceptions and survival: do global evaluations of health status really predict mortality? J Gerontol 1991; 46: S55-S65.

27 Hulsmann M, Berger R, Sturm B, et al. Prediction of outcome by neurohumoral activation, the six-minute walk test and the Minnesota Living with Heart Failure Questionnaire in an outpatient cohort with congestive heart failure. Eur Heart J 2002; 23: 886-891.

28 Kawut SM, Horn EM, Berekashvili KK, et al. New predictors of outcome in idiopathic pulmonary arterial hypertension. Am J Cardiol 2005; 95: 199-203.

29 Simonneau G, Barst RJ, Galie N, et al. Continuous subcutaneous infusion of treprostinil, a prostacyclin analogue, in patients with pulmonary arterial hypertension: a double-blind, randomized, placebo-controlled trial. Am J Respir Crit Care Med 2002; 165: 800-804.

30 Barst RJ, McGoon M, McLaughlin V, et al. Beraprost therapy for pulmonary arterial hypertension. I Am Coll Cardiol 2003; 41: 2119-2125.

31 Archibald CJ, Auger WR, Fedullo PF. Outcome after pulmonary thrombendarterectomy. Semin Thorac Cardiovasc Surg 1999; 11: 164-171. 